\title{
SOLUTIONS WITH SNAKING SINGULARITIES FOR THE FAST DIFFUSION EQUATION
}

\author{
MAREK FILA, JOHN ROBERT KING, JIN TAKAHASHI, AND EIJI YANAGIDA
}

\begin{abstract}
We construct solutions of the fast diffusion equation, which exist for all $t \in \mathbb{R}$ and are singular on the set $\Gamma(t):=\{\xi(s) ;-\infty<s \leq c t\}, c>0$, where $\xi \in C^{3}\left(\mathbb{R} ; \mathbb{R}^{n}\right), n>2$. We also give a precise description of the behavior of the solutions near $\Gamma(t)$.
\end{abstract}

\section{INTRODUCTION}

We study positive singular solutions of the fast diffusion equation

$$
u_{t}=\Delta u^{m}
$$

in $\mathbb{R}^{n}$, where $0<m<1$ and $n \geq 2$. Let $\Gamma$ be a curve in $\mathbb{R}^{n}$ expressed as $\Gamma=\{\xi(s) ; s \in \mathbb{R}\}$ with $\xi: \mathbb{R} \rightarrow \mathbb{R}^{n}$. We are interested in a positive entire-in-time solution that is singular on the set

$$
\Gamma(t):=\{\xi(s) ;-\infty<s \leq c t\} \subset \Gamma \quad \text { for each } t \in \mathbb{R},
$$

where $c>0$ is a constant. Such a solution can be called a snaking solution (or a solution with a snaking singularity). We first introduce our result and then give a brief survey about some different singular solutions.

For $x \in \mathbb{R}^{n}$ and $r_{0}>0$, we write

$$
r(x):=\operatorname{dist}(x, \Gamma), \quad \Gamma_{r_{0}}:=\left\{x \in \mathbb{R}^{n} ; 0 \leq r(x)<r_{0}\right\},
$$

where $\operatorname{dist}(x, \Gamma):=\inf _{y \in \Gamma}|x-y|$. In what follows, we always impose the following condition.

Condition 1.1. $\Gamma$ is a curve expressed as $\Gamma=\{\xi(s) ; s \in \mathbb{R}\}$. Here $\xi \in C^{3}\left(\mathbb{R} ; \mathbb{R}^{n}\right)$ is an injection satisfying $\left|\xi^{\prime}\right| \equiv 1$. Moreover, $\xi$ satisfies the following:

(i) There exists a constant $K>1$ such that $\left|\xi^{\prime \prime}(s)\right|,\left|\xi^{\prime \prime \prime}(s)\right| \leq K$ for all $s \in \mathbb{R}$.

(ii) There exists a constant $0<\tilde{r}_{0}<(2 K)^{-1}$ such that, for any $x \in \Gamma_{\tilde{r}_{0}}$, there exists a unique number $s(x) \in \mathbb{R}$ satisfying $r(x)=|x-\xi(s(x))|$.

Set

$$
Q:=\left\{(x, t) \in \mathbb{R}^{n+1} ; x \in \mathbb{R}^{n} \backslash \Gamma(t), t \in \mathbb{R}\right\}
$$

1991 Mathematics Subject Classification. Primary 35K67; Secondary 35A21, 35B40.

The first author was supported in part by the Slovak Research and Development Agency under the contract No. APVV-18-0308 and by VEGA grant 1/0347/18. The third author was supported in part by JSPS KAKENHI Early-Career Scientists (No. 19K14567). The fourth author was supported in part by JSPS KAKENHI Grant-in-Aid for Scientific Research (A) (No. JP17H01095). 
We define an exponent $m_{*}$ by

$$
m_{*}:= \begin{cases}0 & \text { if } n=2, \\ \frac{n-3}{n-1} & \text { if } n \geq 3 .\end{cases}
$$

Our main result implies the existence of a solution with a snaking singularity if $m_{*}<m<1$.

Theorem 1.2. Let $n \geq 2, m_{*}<m<1, c>0$ and $0<\varepsilon<1$. Assume that $\Gamma$ satisfies Condition 1.1. Then there exists a positive solution $u \in C^{2,1}(Q)$ of $(1.1)$ in $Q$ such that the following estimate holds. There exists a constant $0<\delta<\tilde{r}_{0}$ depending on $c$ and $\varepsilon$ such that

$$
(1-\varepsilon) U(x, t) \leq u(x, t) \leq(1+\varepsilon) U(x, t)
$$

for any $(x, t) \in \mathbb{R}^{n+1}$ with $0<r(x) \leq \delta$ and $-\infty<s(x) \leq c t+\delta$, where

$$
\begin{aligned}
U(x, t):= & c^{-\frac{1}{1-m}}\left(\frac{(n-1) m}{1-m}\left(m-\frac{n-3}{n-1}\right)\right)^{\frac{1}{1-m}} \\
& \times\left(\sqrt{r^{2}(x)+(s(x)-c t)^{2}}+s(x)-c t\right)^{-\frac{1}{1-m}} .
\end{aligned}
$$

In particular, $u$ is singular on $\Gamma(t)=\{\xi(s) ;-\infty<s \leq c t\}$ for each $t \in \mathbb{R}$.

Let us now mention some known results on positive singular solutions of the equation

$$
u_{t}=\Delta u^{m}, \quad x \in \mathbb{R}^{n} \backslash\{\theta(t)\}, \quad t>0,
$$

where $0<m<1, n \geq 2$ and $\theta \in C^{1}\left([0, \infty) ; \mathbb{R}^{n}\right)$ is a given function. We consider (1.3) with the initial condition

$$
u(x, 0)=u_{0}(x), \quad x \in \mathbb{R}^{n} \backslash\{\theta(0)\} .
$$

We are interested in solutions that are singular at $\theta(t)$, that is,

$$
u(x, t) \rightarrow \infty \quad \text { as } x \rightarrow \theta(t), \quad t \geq 0 .
$$

For example, when $\theta \equiv 0$ and $n \geq 3,(1.3)$ has a singular steady state given by

$$
\tilde{u}(x)=C|x|^{-\frac{n-2}{m}}, \quad x \in \mathbb{R}^{n} \backslash\{0\},
$$

where $C$ is an arbitrary positive constant. Another explicit singular solution for $\theta \equiv 0, u_{0} \equiv 0$ and

is

$$
m_{c}:=\frac{(n-2)_{+}}{n}<m<1
$$

$$
u^{*}(x, t):=\left(\frac{c t}{|x|^{2}}\right)^{\frac{1}{1-m}}, \quad c:=2 m\left(\frac{2}{1-m}-n\right) .
$$

For $0<m<1$ and $n \geq 2$, one can find in [1] a complete classification of nonnegative solutions of $u_{t}=\Delta u^{m}$ in $\mathcal{D}^{\prime}\left(\left(\mathbb{R}^{n} \backslash\{0\}\right) \times(0, \infty)\right)$ which are continuous in $\mathbb{R}^{n} \times[0, \infty)$ with values in $(0, \infty]$, satisfy $(1.4)$ with $u_{0} \equiv 0$ and $(1.5)$.

For the existence of self-similar solutions with a standing singularity $(\theta \equiv 0)$, we refer to [1] when $m_{c}<m<1$ and to [12,26] when $0<m<m_{c}$ and $n \geq 3$.

If $m_{c}<m<1$ then all weak solutions of $u_{t}=\Delta u^{m}$ with locally integrable initial data $u_{0}$ become immediately bounded and continuous, see [9]. On the other hand, in the same range $m_{c}<m<1$, stronger singularities may persist, see 
$[2,3]$. It was shown in [2] that for $m_{c}<m<1$, the strongly singular set of $u_{0}$ cannot shrink in time for extended continuous solutions. Here the strongly singular set of $u_{0}$ is defined as the set of points at which $u_{0}$ is not locally integrable and an extended continuous solution satisfies the equation pointwise in the set $\left\{(x, t) \in \mathbb{R}^{n+1} ; v(x, t)<\infty\right\}$, and is continuous with values in $(0, \infty]$. The existence of extended continuous solutions with expanding strongly singular sets is also established in [2].

The evolution of standing singularities of proper (minimal) solutions of $u_{t}=$ $\Delta u^{m}$ on a bounded domain was studied in [27] for $n \geq 3$ and $0<m \leq m_{c}$. By a proper solution we mean a solution obtained as a limit of increasing bounded approximations.

It was shown in [6] that for $m>(n-2) /(n-1)$ and $n \geq 3$, under some assumptions on given functions $\theta, k, u_{0}$, there are solutions of (1.3)-(1.5) which behave as $k(t)|x-\theta(t)|^{-(n-2) / m}$ near $x=\theta(t)$. This corresponds to the singularity of the steady state $\tilde{u}$. For $m<(n-2) /(n-1)$ such solutions with moving singularities do not exist. The case $n=2$ has been treated recently in [5]. It has been established there that solutions with moving singularities, which behave near the singularity like the fundamental solution of the Laplace equation, raised to the power $1 / \mathrm{m}$, exist for $m>0$.

For various results on solutions with moving singularities $(\theta \not \equiv 0)$ for the heat equation $(m=1)$ we refer to $[13,14,24]$, for semilinear heat equations see $[10,11$, $14,15,19-23,25]$ and also [16, 17] for the Navier-Stokes system.

Our result is somehow disconnected from the previous ones since the nature of the singularity described here is novel and different. Our aim is to contribute to a deeper understanding of singularity formation and non-uniqueness phenomena for the fast diffusion equation. The implications for the Cauchy problem consist in showing the existence of initial functions from which a strong singularity can move in time along a prescribed curve, leaving the solution singular behind. As far as we know, this kind of behavior has not been observed previously.

The idea of the proof is to use matched asymptotics in order to construct suitable sub- and super-solutions. The most important part of them is derived from an explicit entire solution which can be found in the special case when the curve is a straight line. We also rely on some delicate properties of the distance function. Once entire comparison functions are constructed, the proof can be finished by standard methods.

The paper is organised as follows. In Section 2 we study an explicit solution when $\{\Gamma(t) ; t \in \mathbb{R}\}$ is a straight line. In Section 3 we prepare suitable comparison functions for the proof of Theorem 1.2. Section 4 contains the proof and Section 5 a discussion. In Appendix A we give a short derivation of the formula for the explicit solution from Section 2.

\section{ExPLICIT SOLUTION}

In this section, we consider the case $\xi(s)=s \omega$, where $\omega \in \mathbb{R}^{n}$ is a unit vector.

2.1. Singular traveling wave solution. Let $c>0$ be a constant and let $a:=c \omega$ be a velocity vector. Set $x=y+t a$. Then by taking $v(y, t)=u(y+t a, t)$, we see that $v$ satisfies the equation

$$
v_{t}=\Delta_{y} v^{m}+a \cdot \nabla_{y} v
$$


If $m_{*}<m<1$, this equation has a stationary solution explicitly expressed as

$$
\begin{aligned}
& \varphi=\varphi(y):=A(|a||y|+a \cdot y)^{-\frac{1}{1-m}} \\
& A=A(n, m):=\left(\frac{(n-1) m}{1-m}\left(m-\frac{n-3}{n-1}\right)\right)^{\frac{1}{1-m}} .
\end{aligned}
$$

We observe that the solution

$$
u(x, t)=\varphi(x-t a)=A(|a||x-t a|+a \cdot(x-t a))^{-\frac{1}{1-m}}
$$

of (1.1) has a singularity on the set $\{s \omega ;-\infty<s \leq c t\}$ for each $t \in \mathbb{R}$, and so we call this solution the singular traveling wave solution.

We note that, in the case where $n=1$ and $0<m<1$, the explicit solution in (2.2) is known as a semi-wavefront solution, cf. for instance [8, Section 2.1]. See Appendix A for a straightforward derivation of (2.2)-(2.3).

2.2. Stability of the singular traveling wave solution. By a direct computation, we have

$$
\Delta \varphi^{m}=-a \cdot \nabla \varphi=\frac{A}{1-m} \frac{|a|}{|y|}(|a||y|+a \cdot y)^{-\frac{1}{1-m}} \geq 0 .
$$

Let $0<\gamma<1$. Setting $\varphi^{+}(y):=(1+\gamma) \varphi(y)$ gives

$$
\begin{aligned}
\Delta\left(\varphi^{+}\right)^{m}+a \cdot \nabla \varphi^{+} & =(1+\gamma)^{m} \Delta \varphi^{m}+(1+\gamma) a \cdot \nabla \varphi \\
& =\left((1+\gamma)^{m}-(1+\gamma)\right) \Delta \varphi^{m} \leq 0 .
\end{aligned}
$$

Hence $\varphi^{+}$is a super-solution of $(2.1)$. Similarly, $\varphi^{-}(y):=(1-\gamma) \varphi(y)$ is a subsolution of (2.1), and so the functions

$$
u^{ \pm}(x, t):=\varphi^{ \pm}(x-t a)=(1 \pm \gamma) \varphi(x-t a)
$$

are a super-solution and a sub=solution of (1.1), respectively. Thus, the singular traveling wave solution $\varphi(x-t a)$ is stable, and so it is expected that we can construct suitable comparison functions for proving Theorem 1.2.

2.3. Singular traveling wave solution in cylindrical coordinates. For $x \in$ $\mathbb{R}^{n}$, let $r(x)$ be the distance between $x$ and the line $\{\xi(s)=s \omega ; s \in \mathbb{R}\}$. Writing

$$
x=z(x)+s(x) \omega, \quad z(x) \perp \omega,
$$

we have $r(x)=|z(x)|=|x-s(x) \omega|$ and $s(x)=\omega \cdot x$. Then by using

$$
x-t a=x-s(x) \omega+s(x) \omega-c t \omega=z(x)+(s(x)-c t) \omega,
$$

we obtain

$$
\begin{aligned}
& |a||x-t a|=c \sqrt{|z(x)|^{2}+|(s(x)-c t) \omega|^{2}}=c \sqrt{r^{2}(x)+(s(x)-c t)^{2}}, \\
& a \cdot(x-t a)=c \omega \cdot z(x)+c \omega \cdot(s(x)-c t) \omega=c(s(x)-c t) .
\end{aligned}
$$

Hence the traveling singular solution can be written as

$$
\begin{aligned}
& u(x, t)=\psi(r(x), s(x)-c t)=A c^{-\frac{1}{1-m}}\left(\sqrt{r^{2}(x)+(s(x)-c t)^{2}}+s(x)-c t\right)^{-\frac{1}{1-m}}, \\
& \psi=\psi(r, \sigma):=A c^{-\frac{1}{1-m}}\left(\sqrt{r^{2}+\sigma^{2}}+\sigma\right)^{-\frac{1}{1-m}} .
\end{aligned}
$$

Based on the above observation, we handle more general cases in the subsequent sections. 


\section{Comparison FUnCtions}

In what follows, we take $r_{0}>0$ such that $0<r_{0}<\tilde{r}_{0}$, where $\tilde{r}_{0}(<1)$ is given in Condition 1.1. Then for any $x \in \Gamma_{r_{0}}$, there exists a unique real number $s(x) \in \mathbb{R}$ such that $r(x)=|x-\xi(s(x))|$. We remark that $r(x)$ and $s(x)$ are $C^{3}$-functions on $\Gamma_{r_{0}} \backslash \Gamma$, since $\xi$ is $C^{3}$.

The goal of this section is to prove the following proposition, which guarantees the existence of suitable comparison functions for showing Theorem 1.2.

Proposition 3.1. Let $n \geq 2, m_{*}<m<1, c>0$ and $0<\varepsilon<1$. Then there exist a super-solution $\bar{u}$ and a sub-solution $\underline{u}$ of (1.1) in $Q$ such that the following (i) and (ii) hold.

(i) $\bar{u} \geq \underline{u}>0$ on $Q$.

(ii) There exists a constant $0<\delta<r_{0}$ depending on $c$ and $\varepsilon$ such that

$$
(1-\varepsilon) U(x, t) \leq \underline{u}(x, t) \leq \bar{u}(x, t) \leq(1+\varepsilon) U(x, t)
$$

for any $(x, t) \in \mathbb{R}^{n+1}$ satisfying $0<r(x) \leq \delta$ and $-\infty<s(x) \leq c t+\delta$, where $U$ is given in Theorem 1.2.

3.1. Ingredients of comparison functions. Our comparison functions are based on the following function.

$$
\begin{aligned}
& U(x, t)=\psi(r(x), \sigma(x, t))=A c^{-\frac{1}{1-m}}\left(\sqrt{r^{2}(x)+\sigma^{2}(x, t)}+\sigma(x, t)\right)^{-\frac{1}{1-m}}, \\
& \sigma(x, t):=s(x)-c t
\end{aligned}
$$

where $U$ is the same as in Theorem 1.2. Notice that $U$ is defined at least on the set

$$
\left\{(x, t) \in \mathbb{R}^{n+1} ; \begin{array}{l}
\text { either } x \in \Gamma_{\tilde{r}_{0}} \backslash \Gamma \text { with } s(x) \leq c t, \\
\text { or } x \in \Gamma_{\tilde{r}_{0}} \text { with } s(x)>c t
\end{array}\right\},
$$

where $\tilde{r}_{0}$ is given in Condition 1.1. We observe that $U(\cdot, t)$ is singular on the set $\Gamma(t)=\{\xi(s) ;-\infty<s \leq c t\}$ for each $t \in \mathbb{R}$. In order to compute the derivatives of $U$, we explicitly compute $\nabla s, \nabla r, \Delta s$ and $\Delta r$ as follows. First, we prepare a fundamental lemma.

Lemma 3.2. For $x \in \Gamma_{r_{0}} \backslash \Gamma$, the following equality holds.

$$
(x-\xi(s(x))) \cdot \xi^{\prime}(s(x))=0 .
$$

Moreover, $\xi^{\prime}(s(x)) \cdot \xi^{\prime \prime}(s(x))=0$ also holds for $x \in \Gamma_{r_{0}} \backslash \Gamma$.

Proof. Since $\xi(s(x))$ is the nearest point from $x$, we have

$$
\begin{aligned}
& \left.\partial_{s}\left(|x-\xi(s)|^{2}\right)\right|_{s=s(x)}=-2(x-\xi(s(x))) \cdot \xi^{\prime}(s(x))=0, \\
& \left.\partial_{s}^{2}\left(|x-\xi(s)|^{2}\right)\right|_{s=s(x)}=2\left|\xi^{\prime}(s(x))\right|^{2}-2(x-\xi(s(x))) \cdot \xi^{\prime \prime}(s(x)) \geq 0 .
\end{aligned}
$$

Then (3.1) follows. Moreover, by $\left|\xi^{\prime}(s(x))\right|^{2}=1$, we also have $(x-\xi(s(x)))$. $\xi^{\prime \prime}(s(x)) \leq 1$. From $\left|\xi^{\prime}(s)\right|^{2}=1$ for $s \in \mathbb{R}$, it follows that $\xi^{\prime}(s) \cdot \xi^{\prime \prime}(s)=0$ for $s \in \mathbb{R}$.

Note that $\left|(x-\xi(s(x))) \cdot \xi^{\prime \prime}(s(x))\right| \leq r_{0} K \leq 1 / 2$ by $r_{0}<\tilde{r}_{0}$ and Condition 1.1. By using this lemma, we can compute $\nabla s, \nabla r, \Delta s$ and $\Delta r$ as follows. 
Lemma 3.3. For $x \in \Gamma_{r_{0}} \backslash \Gamma$, the following equalities hold.

$$
\begin{aligned}
\nabla s(x) & =\frac{\xi^{\prime}(s(x))}{1-(x-\xi(s(x))) \cdot \xi^{\prime \prime}(s(x))}, \\
\nabla r(x) & =\frac{x-\xi(s(x))}{|x-\xi(s(x))|}, \\
\Delta s(x) & =\frac{(x-\xi(s(x))) \cdot \xi^{\prime \prime \prime}(s(x))}{\left[1-(x-\xi(s(x))) \cdot \xi^{\prime \prime}(s(x))\right]^{3}}, \\
\Delta r(x) & =\frac{n-2-(n-1)(x-\xi(s(x))) \cdot \xi^{\prime \prime}(s(x))}{1-(x-\xi(s(x))) \cdot \xi^{\prime \prime}(s(x))} r(x)^{-1} .
\end{aligned}
$$

In particular, $\nabla s(x) \cdot \nabla r(x)=0$.

Proof. In this proof, we write $s=s(x)$ and $r=r(x)$ for short. From the differentiation of (3.1) with respect to $x$, it follows that

$$
\begin{aligned}
0 & =\xi^{\prime}(s)-\left|\xi^{\prime}(s)\right|^{2} \nabla s+\left[(x-\xi(s)) \cdot \xi^{\prime \prime}(s)\right] \nabla s \\
& =\xi^{\prime}(s)-\nabla s+\left[(x-\xi(s)) \cdot \xi^{\prime \prime}(s)\right] \nabla s .
\end{aligned}
$$

Then,

$$
\nabla s=\frac{\xi^{\prime}(s)}{1-(x-\xi(s)) \cdot \xi^{\prime \prime}(s)} .
$$

By taking the divergence in (3.2), we have

$$
\begin{aligned}
& \xi^{\prime \prime}(s) \cdot \nabla s-\Delta s+\xi^{\prime \prime}(s) \cdot \nabla s-\xi^{\prime}(s) \cdot \xi^{\prime \prime}(s)|\nabla s|^{2} \\
& +(x-\xi(s)) \cdot \xi^{\prime \prime \prime}(s)|\nabla s|^{2}+\left[(x-\xi(s)) \cdot \xi^{\prime \prime}(s)\right] \Delta s=0 .
\end{aligned}
$$

This together with $\xi^{\prime} \cdot \xi^{\prime \prime} \equiv 0$ shows that

$$
-\Delta s+(x-\xi(s)) \cdot \xi^{\prime \prime \prime}(s)|\nabla s|^{2}+\left[(x-\xi(s)) \cdot \xi^{\prime \prime}(s)\right] \Delta s=0,
$$

and so

$$
\Delta s=\frac{(x-\xi(s)) \cdot \xi^{\prime \prime \prime}(s)}{1-(x-\xi(s)) \cdot \xi^{\prime \prime}(s)}|\nabla s|^{2}=\frac{(x-\xi(s)) \cdot \xi^{\prime \prime \prime}(s)}{\left[1-(x-\xi(s)) \cdot \xi^{\prime \prime}(s)\right]^{3}} .
$$

By the differentiation of $r=|x-\xi(s)|$ and by (3.1), we have

$$
\nabla r=\frac{x-\xi(s)-\left[(x-\xi(s)) \cdot \xi^{\prime}(s)\right] \nabla s}{|x-\xi(s)|}=\frac{x-\xi(s)}{|x-\xi(s)|} .
$$

By (3.1) again, we also have $\nabla s \cdot \nabla r=0$. Taking the divergence of $\nabla r=(x-$ $\xi(s)) r^{-1}$ yields

$$
\begin{aligned}
\Delta r & =n r^{-1}-r^{-1} \xi^{\prime}(s) \cdot \nabla s-r^{-2}(x-\xi(s)) \cdot \nabla r \\
& =\left[n-1-\xi^{\prime}(s) \cdot \nabla s\right] r^{-1} \\
& =\left[n-1-\frac{\left|\xi^{\prime}(s)\right|^{2}}{1-(x-\xi(s)) \cdot \xi^{\prime \prime}(s)}\right] r^{-1} .
\end{aligned}
$$

This together with $\left|\xi^{\prime}\right| \equiv 1$ shows the desired equality for $\Delta r$.

From Lemma 3.3 and Condition 1.1, the following lemma immediately follows. 
Lemma 3.4. For $x \in \Gamma_{r_{0}} \backslash \Gamma$, the following inequalities hold.

$$
\begin{aligned}
& 2^{-1} \leq\left(1+r_{0} K\right)^{-1} \leq|\nabla s(x)| \leq\left(1-r_{0} K\right)^{-1} \leq 2, \\
& -8 r_{0} K \leq-\frac{r_{0} K}{\left(1-r_{0} K\right)^{3}} \leq \Delta s(x) \leq \frac{r_{0} K}{\left(1-r_{0} K\right)^{3}} \leq 8 r_{0} K, \\
& \frac{n-2-(n-1) r_{0} K}{1+r_{0} K} r(x)^{-1} \leq \Delta r(x) \leq \frac{n-2+(n-1) r_{0} K}{1-r_{0} K} r(x)^{-1} .
\end{aligned}
$$

We next compute the derivatives of $U$. Set

$$
Q_{r_{0}}:=\left\{(x, t) \in \mathbb{R}^{n+1} ; \quad \begin{array}{l}
\text { either } x \in \Gamma_{r_{0}} \backslash \Gamma \text { with } s(x) \leq c t \\
\text { or } x \in \Gamma_{r_{0}} \text { with } s(x)>c t
\end{array}\right\} .
$$

Lemma 3.5. For $(x, t) \in Q_{r_{0}}$, the following equalities hold.

$$
\begin{aligned}
& U_{t}=\frac{1}{1-m}\left(r^{2}+\sigma^{2}\right)^{-\frac{1}{2}} c U, \\
& \begin{aligned}
\Delta U^{m}= & {\left[\frac{A^{m-1} m}{(1-m)^{2}}\left(r^{2}+\sigma^{2}\right)^{-\frac{1}{2}} c U\right]\left[-\left(1-|\nabla s(x)|^{2}\right)\left(\frac{(1-m) \sigma^{2}}{r^{2}+\sigma^{2}}+\frac{\sigma}{\sqrt{r^{2}+\sigma^{2}}}\right)\right.} \\
& \left.+\left(1+m|\nabla s(x)|^{2}\right)-(1-m) r \Delta r(x)-(1-m)\left(\sqrt{r^{2}+\sigma^{2}}+\sigma\right) \Delta s(x)\right],
\end{aligned}
\end{aligned}
$$

where $r=r(x)$ and $\sigma=\sigma(x, t)=s(x)-c t$.

Proof. The time derivative $U_{t}$ is easy. The spatial derivative $\Delta U^{m}$ is computed as

$$
\Delta U^{m}=\left(\psi^{m}\right)_{r r}|\nabla r(x)|^{2}+\left(\psi^{m}\right)_{\sigma \sigma}|\nabla s(x)|^{2}+\left(\psi^{m}\right)_{r} \Delta r(x)+\left(\psi^{m}\right)_{\sigma} \Delta s(x) .
$$

Straightforward computations show that

$$
\begin{aligned}
\left(\psi^{m}\right)_{r} & =-\frac{A^{m-1} m}{1-m} r\left(r^{2}+\sigma^{2}\right)^{-\frac{1}{2}} c U, \\
\left(\psi^{m}\right)_{r r} & =\left[\frac{A^{m-1} m}{(1-m)^{2}}\left(1-\frac{\sigma}{\sqrt{r^{2}+\sigma^{2}}}\right)-\frac{A^{m-1} m}{1-m} \frac{\sigma^{2}}{r^{2}+\sigma^{2}}\right]\left(r^{2}+\sigma^{2}\right)^{-\frac{1}{2}} c U, \\
\left(\psi^{m}\right)_{\sigma} & =-\frac{A^{m-1} m}{1-m}\left(\sqrt{r^{2}+\sigma^{2}}+\sigma\right)\left(r^{2}+\sigma^{2}\right)^{-\frac{1}{2}} c U, \\
\left(\psi^{m}\right)_{\sigma \sigma} & =\left[\frac{A^{m-1} m}{(1-m)^{2}}\left(1+\frac{\sigma}{\sqrt{r^{2}+\sigma^{2}}}\right)-\frac{A^{m-1} m}{1-m} \frac{r^{2}}{r^{2}+\sigma^{2}}\right]\left(r^{2}+\sigma^{2}\right)^{-\frac{1}{2}} c U .
\end{aligned}
$$

From these equalities and $|\nabla r|=1$, the desired equality for $\Delta U^{m}$ follows.

3.2. Super-solution near $\Gamma$. Fix $0<\varepsilon^{\prime}<1$. Set

$$
u^{+}(x, t):=\left(1+\varepsilon^{\prime}\right)\left(U^{m}(x, t)+1\right)^{\frac{1}{m}} .
$$

We check that $u^{+}$is a super-solution of (1.1) on $Q_{r_{0}}$ provided that $r_{0}$ is small enough. By Lemma 3.5, we have

$$
\begin{aligned}
u_{t}^{+} & =\left(1+\varepsilon^{\prime}\right)\left(1+U^{-m}\right)^{\frac{1}{m}-1} U_{t} \\
& =\frac{1+\varepsilon^{\prime}}{1-m}\left(1+U^{-m}\right)^{\frac{1-m}{m}}\left(r^{2}+\sigma^{2}\right)^{-\frac{1}{2}} c U \\
& =\frac{1+\varepsilon^{\prime}}{1-m}\left(1+A^{-m} c^{\frac{m}{1-m}}\left(\sqrt{r^{2}+\sigma^{2}}+\sigma\right)^{\frac{m}{1-m}}\right)^{\frac{1-m}{m}}\left(r^{2}+\sigma^{2}\right)^{-\frac{1}{2}} c U
\end{aligned}
$$


Since $\Delta\left(u^{+}\right)^{m}=\left(1+\varepsilon^{\prime}\right)^{m} \Delta U^{m}$, we will estimate $\Delta U^{m}$. By Lemmas 3.5 and 3.4 , we have

$$
\begin{aligned}
\Delta U^{m} \leq & {\left[\frac{A^{m-1} m}{(1-m)^{2}}\left(r^{2}+\sigma^{2}\right)^{-\frac{1}{2}} c U\right]\left[\left.|1-| \nabla s(x)\right|^{2} \mid\left(\frac{(1-m) \sigma^{2}}{r^{2}+\sigma^{2}}+\frac{|\sigma|}{\sqrt{r^{2}+\sigma^{2}}}\right)\right.} \\
& +1+\frac{m}{\left(1-r_{0} K\right)^{2}}-(1-m) \frac{n-2-(n-1) r_{0} K}{1+r_{0} K} \\
& \left.+8 r_{0} K(1-m)\left(\sqrt{r^{2}+\sigma^{2}}+\sigma\right)\right] .
\end{aligned}
$$

From the estimate of $|\nabla s|$ in Lemma 3.4, (2.3) and

$$
\frac{(1-m) \sigma^{2}}{r^{2}+\sigma^{2}}+\frac{|\sigma|}{\sqrt{r^{2}+\sigma^{2}}} \leq 2
$$

it follows that

$$
\begin{aligned}
\Delta U^{m} \leq & {\left[\frac{A^{m-1} m(n-1)}{(1-m)^{2}}\left(m-\frac{n-3}{n-1}\right)\right.} \\
& \left.+\frac{A^{m-1} m}{(1-m)^{2}} 8 r_{0} K(1-m)\left(\sqrt{r^{2}+\sigma^{2}}+\sigma\right)+O\left(r_{0}\right)\right]\left(r^{2}+\sigma^{2}\right)^{-\frac{1}{2}} c U \\
\leq & {\left[\frac{1}{1-m}+C r_{0}\left(\sqrt{r^{2}+\sigma^{2}}+\sigma\right)+O\left(r_{0}\right)\right]\left(r^{2}+\sigma^{2}\right)^{-\frac{1}{2}} c U }
\end{aligned}
$$

with a constant $C=C(m, A, K)>0$ as $r_{0} \rightarrow 0$. Thus,

$$
\Delta\left(u^{+}\right)^{m} \leq\left[\frac{\left(1+\varepsilon^{\prime}\right)^{m}}{1-m}+C r_{0}\left(\sqrt{r^{2}+\sigma^{2}}+\sigma\right)+O\left(r_{0}\right)\right]\left(r^{2}+\sigma^{2}\right)^{-\frac{1}{2}} c U
$$

with a constant $C=C\left(m, A, K, \varepsilon^{\prime}\right)>0$ as $r_{0} \rightarrow 0$.

The above computations show that

$$
\begin{aligned}
u_{t}^{+}-\Delta\left(u^{+}\right)^{m} \geq & \frac{\left(1+\varepsilon^{\prime}\right)^{m}}{1-m}\left[\left(1+\varepsilon^{\prime}\right)^{1-m}\left(1+A^{-m} c^{\frac{m}{1-m}}\left(\sqrt{r^{2}+\sigma^{2}}+\sigma\right)^{\frac{m}{1-m}}\right)^{\frac{1-m}{m}}\right. \\
& \left.-1-C r_{0}\left(\sqrt{r^{2}+\sigma^{2}}+\sigma\right)+O\left(r_{0}\right)\right]\left(r^{2}+\sigma^{2}\right)^{-\frac{1}{2}} c U
\end{aligned}
$$

We first assume that

$$
A^{-m} c^{\frac{m}{1-m}}\left(\sqrt{r^{2}+\sigma^{2}}+\sigma\right)^{\frac{m}{1-m}} \geq 2 .
$$

Then,

$$
\begin{aligned}
& \left(1+\varepsilon^{\prime}\right)^{1-m}\left(1+A^{-m} c^{\frac{m}{1-m}}\left(\sqrt{r^{2}+\sigma^{2}}+\sigma\right)^{\frac{m}{1-m}}\right)^{\frac{1-m}{m}}-1-C r_{0}\left(\sqrt{r^{2}+\sigma^{2}}+\sigma\right) \\
& \geq\left(1+\varepsilon^{\prime}\right)^{1-m} A^{-(1-m)} c\left(\sqrt{r^{2}+\sigma^{2}}+\sigma\right)-1-C r_{0}\left(\sqrt{r^{2}+\sigma^{2}}+\sigma\right) \\
& \geq 2^{\frac{1}{m}}\left(1+\varepsilon^{\prime}\right)^{1-m}-1+\left(\frac{\left(1+\varepsilon^{\prime}\right)^{1-m}}{2} A^{-(1-m)} c-C r_{0}\right)\left(\sqrt{r^{2}+\sigma^{2}}+\sigma\right) \geq 0
\end{aligned}
$$

provided that $r_{0}$ is small depending on $\varepsilon^{\prime}, m, c$ and $A$. On the other hand, we next assume that

$$
A^{-m} c^{\frac{m}{1-m}}\left(\sqrt{r^{2}+\sigma^{2}}+\sigma\right)^{\frac{m}{1-m}} \leq 2 \text {. }
$$


In this case, we have

$$
\begin{aligned}
& \left(1+\varepsilon^{\prime}\right)^{1-m}\left(1+A^{-m} c^{\frac{m}{1-m}}\left(\sqrt{r^{2}+\sigma^{2}}+\sigma\right)^{\frac{m}{1-m}}\right)^{\frac{1-m}{m}}-1-C r_{0}\left(\sqrt{r^{2}+\sigma^{2}}+\sigma\right) \\
& \geq\left(1+\varepsilon^{\prime}\right)^{1-m}-1-2^{\frac{1-m}{m}} C A^{1-m} c^{-1} r_{0} \geq 0
\end{aligned}
$$

provided that $r_{0}$ is small depending on $m, A, c$ and $C=C\left(m, A, K, \varepsilon^{\prime}\right)$. Hence $u^{+}$ is a super-solution in $Q_{r_{0}}$ provided that $r_{0}$ is sufficiently small depending only on $m, A, K, c$ and $\varepsilon^{\prime}$.

3.3. Super-solution on $Q$. We construct a super-solution on $Q$, where $Q$ is defined by (1.2). For $B, b>1$, define

$$
\bar{u}(x, t):= \begin{cases}{\left[\eta(r(x))\left(u^{+}\right)^{m}(x, t)+B(b-\eta(r(x)))\right]^{\frac{1}{m}}} & \text { if } r(x) \leq r_{0}, \\ (B b)^{\frac{1}{m}} & \text { otherwise. }\end{cases}
$$

Here $\eta$ is defined below. Let $r_{1}$ and $r_{2}$ satisfy $0<r_{1}<r_{2}<r_{0}$. We define $\eta \in C^{\infty}([0, \infty))$ by

$$
\eta=\eta(\rho):= \begin{cases}1 & \text { for } 0 \leq \rho \leq r_{1}, \\ \frac{\Phi(\rho)}{\Phi(\rho)+\Psi(\rho)} & \text { for } r_{1}<\rho<r_{2}, \\ 0 & \text { for } \rho \geq r_{2},\end{cases}
$$

where

$$
\Phi(\rho):=\exp \left(-\frac{1}{r_{2}-\rho}\right), \quad \Psi(\rho):=\exp \left(-\frac{1}{\rho-r_{1}}\right) .
$$

After straightforward computations, we have

$$
\begin{aligned}
\Phi^{\prime} & =-\frac{1}{\left(r_{2}-\rho\right)^{2}} \Phi, \quad \Phi^{\prime \prime}=\frac{1-2 r_{2}+2 \rho}{\left(r_{2}-\rho\right)^{4}} \Phi, \\
\Psi^{\prime} & =\frac{1}{\left(\rho-r_{1}\right)^{2}} \Psi, \quad \Psi^{\prime \prime}=\frac{1+2 r_{1}-2 \rho}{\left(\rho-r_{1}\right)^{4}} \Psi, \quad \eta^{\prime}=\frac{\Phi^{\prime} \Psi-\Phi \Psi^{\prime}}{(\Phi+\Psi)^{2}} \\
\eta^{\prime \prime} & =\frac{1}{(\Phi+\Psi)^{2}}\left(\Phi^{\prime \prime} \Psi-\Phi \Psi^{\prime \prime}\right)-\frac{2}{(\Phi+\Psi)^{3}}\left(\Phi^{\prime} \Psi-\Phi \Psi^{\prime}\right)\left(\Phi^{\prime}+\Psi^{\prime}\right),
\end{aligned}
$$

and so

$$
\begin{aligned}
\eta^{\prime}(\rho)= & -\frac{\Phi \Psi}{(\Phi+\Psi)^{2}}\left(\frac{1}{\left(r_{2}-\rho\right)^{2}}+\frac{1}{\left(\rho-r_{1}\right)^{2}}\right) \\
\eta^{\prime \prime}(\rho)= & \frac{\Phi \Psi}{(\Phi+\Psi)^{2}}\left(\frac{1-2 r_{2}+2 \rho}{\left(r_{2}-\rho\right)^{4}}-\frac{1+2 r_{1}-2 \rho}{\left(\rho-r_{1}\right)^{4}}\right) \\
& -\frac{2 \Phi \Psi}{(\Phi+\Psi)^{3}}\left(\frac{1}{\left(r_{2}-\rho\right)^{2}}+\frac{1}{\left(\rho-r_{1}\right)^{2}}\right)\left(\frac{\Phi}{\left(r_{2}-\rho\right)^{2}}-\frac{\Psi}{\left(\rho-r_{1}\right)^{2}}\right)
\end{aligned}
$$

for $r_{1}<\rho<r_{2}$. We note that

$$
\lim _{\rho \uparrow r_{2}} \frac{\eta(\rho)}{\eta^{\prime \prime}(\rho)}=\lim _{\rho \uparrow r_{2}} \frac{\left|\eta^{\prime}(\rho)\right|}{\eta^{\prime \prime}(\rho)}=0 .
$$


By direct computations, we have

$$
\begin{aligned}
& \bar{u}_{t}=\left[\left(u^{+}\right)^{m} \eta+B(b-\eta)\right]^{\frac{1-m}{m}}\left(u^{+}\right)^{m-1} \eta u_{t}^{+}, \\
& \begin{aligned}
-\Delta \bar{u}^{m} & =\left(B-\left(u^{+}\right)^{m}\right) \Delta(\eta(r(x)))-2 \nabla(\eta(r(x))) \cdot \nabla\left(u^{+}\right)^{m}-\eta(r(x)) \Delta\left(u^{+}\right)^{m} \\
& =\left(B-\left(u^{+}\right)^{m}\right)\left(\eta^{\prime \prime}+\eta^{\prime} \Delta r\right)-2 \eta^{\prime} \nabla r \cdot \nabla\left(u^{+}\right)^{m}-\eta \Delta\left(u^{+}\right)^{m},
\end{aligned}
\end{aligned}
$$

where $\nabla r$ and $\Delta r$ are evaluated at $r(x)$. In the region $r(x) \leq r_{1}$, we have

$\bar{u}_{t}-\Delta \bar{u}^{m}=\left[\left(u^{+}\right)^{m}+B(b-1)\right]^{\frac{1-m}{m}}\left(u^{+}\right)^{m-1} u_{t}^{+}-\Delta\left(u^{+}\right)^{m} \geq u_{t}^{+}-\Delta\left(u^{+}\right)^{m} \geq 0$,

since $u_{t}^{+}>0$ by (3.3). On the other hand, in the region $r(x) \geq r_{2}$, we have $\bar{u}_{t}-\Delta \bar{u}^{m}=0$. Hence it suffices to consider the region $r_{1}<r(x)<\bar{r}_{2}$.

First, we observe the case where $r(x)$ is smaller than $r_{2}$ and is close to $r_{2}$. By $u_{t}^{+}>0$, we have $\bar{u}_{t} \geq 0$, and so

$$
\bar{u}_{t}-\Delta \bar{u}^{m} \geq\left(B-\left(u^{+}\right)^{m}\right)\left(\eta^{\prime \prime}+\eta^{\prime} \Delta r\right)-2 \eta^{\prime} \nabla r \cdot \nabla\left(u^{+}\right)^{m}-\eta \Delta\left(u^{+}\right)^{m} .
$$

We take constants $\tilde{C}>1$ and $B$ such that $\tilde{C}^{-1} \leq u^{+} \leq \tilde{C}$ for $r_{1}<r(x)<r_{2}$ and $B>\tilde{C}^{m}$. By (3.6) and $\eta^{\prime \prime}>0$ near $r_{2}$, we have $\eta^{\prime \prime}-\left|\eta^{\prime}\right||\Delta r|>0$ near $r_{2}$. Then there exists a constant $C>1$ independent of $b$ such that

$$
\bar{u}_{t}-\Delta \bar{u}^{m} \geq\left(B-\tilde{C}^{m}\right)\left(\eta^{\prime \prime}-C\left|\eta^{\prime}\right|\right)-C\left|\eta^{\prime}\right|-C \eta .
$$

Then by (3.6) again, there exists a constant $r_{1}<r_{2}^{\prime}<r_{2}$ independent of $b$ such that $\bar{u}_{t}-\Delta(\bar{u})^{m} \geq 0$ for $r_{2}^{\prime}<r(x)<r_{2}$.

We next examine the case where $r_{1}<r(x)<r_{2}^{\prime}$. Note that there exists a constant $\tilde{c}>0$ such that $\tilde{c} \leq \eta(r(x)) \leq 1$ for $r_{1}<r(x)<r_{2}^{\prime}$. By $u_{t}^{+}>0$, there exists a constant $C>1$ depending on $r_{1}$ and $r_{2}$ but not on $b$ such that

$$
\begin{aligned}
& \bar{u}_{t} \geq B^{\frac{1-m}{m}}(b-\eta(r(x)))^{\frac{1-m}{m}}\left(u^{+}\right)^{m-1} \eta u_{t}^{+} \geq \tilde{c} C^{-1} B^{\frac{1-m}{m}}(b-1)^{\frac{1-m}{m}}, \\
& -\Delta \bar{u}^{m}=-\eta \Delta\left(u^{+}\right)^{m}+\left(B-\left(u^{+}\right)^{m}\right)\left(\eta^{\prime \prime}+\eta^{\prime} \Delta r\right)-2 \eta^{\prime} \nabla r \cdot \nabla\left(u^{+}\right)^{m} \geq-C-B C .
\end{aligned}
$$

Then there exists $b>1$ such that $\bar{u}_{t}-\Delta \bar{u}^{m}>0$ for $r_{1}<r(x)<r_{2}^{\prime}$. Hence $\bar{u}$ is a super-solution on $Q$.

3.4. Sub-solution on $Q$. For $M>0$, set

$$
u^{-}(x, t):= \begin{cases}\left(1-\varepsilon^{\prime}\right)\left[U^{m}(x, t)-M-M|\sigma(x, t)|^{\frac{m}{1-m}} \zeta(\sigma(x, t))\right]_{+}^{\frac{1}{m}} & \text { if } r(x) \leq r_{0}, \\ 0 & \text { otherwise, }\end{cases}
$$

where $\sigma(x, t)=s(x)-c t,[\cdot]_{+}$is the positive part and $\zeta \in C^{\infty}(\mathbb{R})$ is a decreasing function satisfying $\zeta(\sigma)=1$ if $\sigma \leq-2, \zeta(\sigma)=0$ if $\sigma \geq-1$ and $0 \leq \zeta \leq 1$. Let $M$ satisfy

$$
M>\max \left\{3^{\frac{m}{1-m}} A^{m} c^{-\frac{m}{1-m}} r_{0}^{-\frac{2 m}{1-m}}, 10^{\frac{m}{1-m}} A^{m} c^{-\frac{m}{1-m}} r_{0}^{-\frac{2 m}{1-m}}\right\} .
$$

We will see that $u^{-}$is a sub-solution of (1.1) on $Q$. By the fact that the maximum of two sub-solutions is also a sub-solution and Lemmas 3.6 and 3.7 below, we only have to consider the case where

$$
(x, t) \in Q_{r_{0}} \cap\left\{u^{-}>0\right\} \quad \text { and } \quad \sigma(x, t) \leq 1 .
$$

Lemma 3.6. If $r(x)=r_{0}$, then $u^{-}(x, t)=0$. 
Proof. Let $x$ satisfy $r(x)=r_{0}$. Then,

$$
\begin{aligned}
U(x, t) & =A c^{-\frac{1}{1-m}}\left(\sqrt{r_{0}^{2}+\sigma^{2}}+\sigma\right)^{-\frac{1}{1-m}} \\
& =A c^{-\frac{1}{1-m}}|\sigma|^{-\frac{1}{1-m}}\left(\sqrt{1+\left(r_{0} / \sigma\right)^{2}}+\sigma /|\sigma|\right)^{-\frac{1}{1-m}} \\
& \leq A c^{-\frac{1}{1-m}}|\sigma|^{-\frac{1}{1-m}}\left(\sqrt{1+\left(r_{0} / \sigma\right)^{2}}-1\right)^{-\frac{1}{1-m}} .
\end{aligned}
$$

We first consider the case $\sigma \leq-2$. From $r_{0} \leq 1$ and $\sigma^{2} \geq 4$, it follows that

$$
\begin{aligned}
\sqrt{1+\left(r_{0} / \sigma\right)^{2}}-1 & =\left(\frac{r_{0}}{\sigma}\right)^{2} \int_{0}^{1} \frac{1}{2}\left(1+\left(\frac{r_{0}}{\sigma}\right)^{2} \theta\right)^{-\frac{1}{2}} d \theta \\
& \geq \frac{1}{2}\left(\frac{r_{0}}{\sigma}\right)^{2}\left(1+\left(\frac{r_{0}}{\sigma}\right)^{2}\right)^{-\frac{1}{2}} \geq \frac{1}{3}\left(\frac{r_{0}}{\sigma}\right)^{2}
\end{aligned}
$$

so that

$$
U(x, t) \leq 3^{\frac{1}{1-m}} A c^{-\frac{1}{1-m}} r_{0}^{-\frac{2}{1-m}}|\sigma|^{\frac{1}{1-m}} .
$$

This together with (3.8) and $\zeta(\sigma)=1$ for $\sigma \leq-2$ gives

$$
\left[U^{m}-M-M|\sigma|^{\frac{m}{1-m}} \zeta(\sigma)\right]_{+} \leq\left[\left(3^{\frac{m}{1-m}} A^{m} c^{-\frac{m}{1-m}} r_{0}^{-\frac{2 m}{1-m}}-M\right)|\sigma|^{\frac{m}{1-m}}\right]_{+}=0 .
$$

We next consider the case $-2 \leq \sigma<0$. From $1 \leq 16 / \sigma^{2}$ and $r_{0}<1$, it follows that

$$
\sqrt{1+\left(r_{0} / \sigma\right)^{2}}-1 \geq \frac{1}{2}\left(\frac{r_{0}}{\sigma}\right)^{2}\left(1+\left(\frac{r_{0}}{\sigma}\right)^{2}\right)^{-\frac{1}{2}} \geq \frac{1}{2}\left(\frac{r_{0}}{\sigma}\right)^{2}\left(\frac{16+r_{0}^{2}}{\sigma^{2}}\right)^{-\frac{1}{2}} \geq \frac{r_{0}^{2}}{10|\sigma|}
$$

By (3.10), we have

$$
U(x, t) \leq 10^{\frac{1}{1-m}} A c^{-\frac{1}{1-m}} r_{0}^{-\frac{2}{1-m}}
$$

and so

$$
\left[U^{m}-M-M|\sigma|^{\frac{m}{1-m}} \zeta(\sigma)\right]_{+} \leq\left[10^{\frac{m}{1-m}} A^{m} c^{-\frac{m}{1-m}} r_{0}^{-\frac{2 m}{1-m}}-M\right]_{+}=0 .
$$

Finally, we examine the case $\sigma \geq 0$. In this case,

$$
\left[U^{m}-M-M|\sigma|^{\frac{m}{1-m}} \zeta(\sigma)\right]_{+} \leq\left[A^{m} c^{-\frac{m}{1-m}} r_{0}^{-\frac{m}{1-m}}-M\right]_{+}=0 .
$$

The lemma follows.

Lemma 3.7. If $\sigma(x, t) \geq 1$, then $u^{-}(x, t)=0$.

Proof. If $\sigma \geq 1$, then

$$
\begin{aligned}
& U(x, t)=A c^{-\frac{1}{1-m}}\left(\sqrt{r^{2}(x)+\sigma^{2}}+\sigma\right)^{-\frac{1}{1-m}} \leq A c^{-\frac{1}{1-m}}, \\
& {\left[U^{m}-M-M|\sigma(x, t)|^{\frac{m}{1-m}} \zeta(\sigma(x, t))\right]_{+} \leq\left[A^{m} c^{-\frac{m}{1-m}}-M\right]_{+}=0,}
\end{aligned}
$$

the lemma follows. 
We consider the case (3.9) with $\sigma \leq-2,-2 \leq \sigma \leq-1$ and $-1 \leq \sigma \leq 1$, respectively. First, we assume (3.9) with $\sigma \leq-2$. By the negativity of $\sigma$ and the positivity of $U_{t}$, we have

$$
\begin{aligned}
u_{t}^{-} & =\left(1-\varepsilon^{\prime}\right) \frac{1}{m}\left[U^{m}-M-M|\sigma|^{\frac{m}{1-m}}\right]^{\frac{1}{m}-1}\left[m U^{m-1} U_{t}+\frac{M m}{1-m} c|\sigma|^{\frac{m}{1-m}-2} \sigma\right] \\
& \leq\left(1-\varepsilon^{\prime}\right) \frac{1}{m} U^{1-m}\left[m U^{m-1} U_{t}+\frac{M m}{1-m} c|\sigma|^{\frac{m}{1-m}-2} \sigma\right] \\
& \leq\left(1-\varepsilon^{\prime}\right) U_{t}=\frac{1-\varepsilon^{\prime}}{1-m}\left(r^{2}+\sigma^{2}\right)^{-\frac{1}{2}} c U .
\end{aligned}
$$

Direct computations yield

$$
\Delta\left(u^{-}\right)^{m}=\left(1-\varepsilon^{\prime}\right)^{m} \Delta U^{m}-\left(1-\varepsilon^{\prime}\right)^{m} M \Delta\left(|\sigma|^{\frac{m}{1-m}}\right) .
$$

By Lemmas 3.5 and 3.4, we have

$$
\begin{aligned}
\Delta U^{m} \geq & {\left[\frac{A^{m-1} m}{(1-m)^{2}}\left(r^{2}+\sigma^{2}\right)^{-\frac{1}{2}} c U\right]\left[-\left.|1-| \nabla s\right|^{2} \mid\left(\frac{(1-m) \sigma^{2}}{r^{2}+\sigma^{2}}+\frac{|\sigma|}{\sqrt{r^{2}+\sigma^{2}}}\right)\right.} \\
& +1+\frac{m}{\left(1+r_{0} K\right)^{2}}-(1-m) \frac{n-2+(n-1) r_{0} K}{1-r_{0} K} \\
& \left.-8 r_{0} K(1-m)\left(\sqrt{r^{2}+\sigma^{2}}+\sigma\right)\right] .
\end{aligned}
$$

From the negativity of $\sigma$, it follows that

$$
\begin{aligned}
\sqrt{r^{2}+\sigma^{2}}+\sigma & =|\sigma|\left(\sqrt{1+(r / \sigma)^{2}}-1\right) \\
& =\frac{r^{2}}{|\sigma|} \int_{0}^{1} \frac{1}{2}\left(1+\left(\frac{r}{|\sigma|}\right)^{2} \theta\right)^{-\frac{1}{2}} d \theta \leq \frac{r^{2}}{2|\sigma|}
\end{aligned}
$$

By $|\sigma| \geq 2$ and $r_{0} \leq 1$, we have

$$
\sqrt{r^{2}+\sigma^{2}}+\sigma \leq \frac{1}{4}
$$

This together with (3.4) implies that

$$
\begin{aligned}
\Delta U^{m} \geq & {\left[\frac{A^{m-1} m}{(1-m)^{2}}\left(r^{2}+\sigma^{2}\right)^{-\frac{1}{2}} c U\right]\left[-2\left(1-\frac{1}{\left(1+r_{0} K\right)^{2}}\right)\right.} \\
& \left.+1+\frac{m}{\left(1+r_{0} K\right)^{2}}-(1-m) \frac{n-2+(n-1) r_{0} K}{1-r_{0} K}-2 r_{0} K(1-m)\right] \\
= & {\left[\frac{A^{m-1} m(n-1)}{(1-m)^{2}}\left(m-\frac{n-3}{n-1}\right)+O\left(r_{0}\right)\right]\left(r^{2}+\sigma^{2}\right)^{-\frac{1}{2}} c U \quad\left(r_{0} \rightarrow 0\right) . }
\end{aligned}
$$

We estimate $\Delta\left(|\sigma|^{\frac{m}{1-m}}\right)$. By computations, we have

$$
\Delta\left(|\sigma|^{\frac{m}{1-m}}\right)=\frac{m}{1-m}\left(\frac{m}{1-m}-1\right)|\sigma|^{\frac{m}{1-m}-2}|\nabla s|^{2}+\frac{m}{1-m}|\sigma|^{\frac{m}{1-m}-2} \sigma \Delta s,
$$

and so

$$
\begin{aligned}
\left|\Delta\left(|\sigma|^{\frac{m}{1-m}}\right)\right| \leq & C\left[\left(r^{2}+\sigma^{2}\right)^{\frac{1}{2}} c^{-1} U^{-1}\left(|\sigma|^{\frac{m}{1-m}-2}|\nabla s|^{2}+|\sigma|^{\frac{m}{1-m}-1}|\Delta s|\right)\right] \\
& \times\left(r^{2}+\sigma^{2}\right)^{-\frac{1}{2}} c U
\end{aligned}
$$


with a constant $C=C(m)>0$. Then, (3.11), $r^{2} \leq \sigma^{2}$, Lemma 3.4 and $|\sigma| \geq 2$ show that

$$
\begin{aligned}
& \left(r^{2}+\sigma^{2}\right)^{\frac{1}{2}} c^{-1} U^{-1}\left(|\sigma|^{\frac{m}{1-m}-2}|\nabla s|^{2}+|\sigma|^{\frac{m}{1-m}-1}|\Delta s|\right) \\
& =\left(r^{2}+\sigma^{2}\right)^{\frac{1}{2}} A^{-1} c^{\frac{1}{1-m}-1}\left(\sqrt{r^{2}+\sigma^{2}}+\sigma\right)^{\frac{1}{1-m}}\left(|\sigma|^{\frac{m}{1-m}-2}|\nabla s|^{2}+|\sigma|^{\frac{m}{1-m}-1}|\Delta s|\right) \\
& \leq C|\sigma|\left(\frac{r_{0}^{2}}{|\sigma|}\right)^{\frac{1}{1-m}}\left(4|\sigma|^{\frac{m}{1-m}-2}+8 r_{0} K|\sigma|^{\frac{m}{1-m}-1}\right) \\
& \leq C r_{0}^{\frac{2}{1-m}}\left(4|\sigma|^{-2}+8 K|\sigma|^{-1}\right) \leq C r_{0}^{\frac{2}{1-m}}(1+4 K)=O\left(r_{0}^{\frac{2}{1-m}}\right) \quad\left(r_{0} \rightarrow 0\right)
\end{aligned}
$$

with some constant $C=C(m, A, c)>0$.

From the above computations and (2.3), it follows that

$$
\begin{aligned}
u_{t}^{-} & -\Delta\left(u^{-}\right)^{m} \\
\leq & {\left[\frac{1-\varepsilon^{\prime}}{1-m}-\left(1-\varepsilon^{\prime}\right)^{m} \frac{A^{m-1} m(n-1)}{(1-m)^{2}}\left(m-\frac{n-3}{n-1}\right)+o(1)\right] } \\
& \times\left(r^{2}+\sigma^{2}\right)^{-\frac{1}{2}} c U \\
= & -\left(1-\varepsilon^{\prime}\right)^{m}\left[\frac{1-\left(1-\varepsilon^{\prime}\right)^{1-m}}{1-m}+o(1)\right]\left(r^{2}+\sigma^{2}\right)^{-\frac{1}{2}} c U \quad\left(r_{0} \rightarrow 0\right) .
\end{aligned}
$$

Hence $u^{-}$is a sub-solution in the case (3.9) with $\sigma \leq-2$ provided that $r_{0}$ is sufficiently small. Remark that the smallness of $r_{0}$ is determined only by $m, n, A$, $c, K, M$ and $\varepsilon^{\prime}$.

Let us next consider the case (3.9) with $-2 \leq \sigma \leq-1$. By the negativity of $\sigma$ and $\zeta^{\prime}$, we have

$$
\begin{aligned}
u_{t}^{-}= & \left(1-\varepsilon^{\prime}\right) \frac{1}{m}\left[U^{m}-M-M|\sigma|^{\frac{m}{1-m}} \zeta(\sigma)\right]^{\frac{1}{m}-1} \\
& \times\left[m U^{m-1} U_{t}+\frac{M m}{1-m} c|\sigma|^{\frac{m}{1-m}-2} \sigma \zeta(\sigma)+M|\sigma|^{\frac{m}{1-m}} c \zeta^{\prime}(\sigma)\right] \leq\left(1-\varepsilon^{\prime}\right) U_{t} .
\end{aligned}
$$

From the smoothness of $|\sigma|^{\frac{m}{1-m}} \zeta(\sigma)$ as a function for $-1 \leq \sigma \leq-2$, it follows that

$$
\begin{aligned}
\Delta\left(u^{-}\right)^{m} & =\left(1-\varepsilon^{\prime}\right)^{m} \Delta U^{m}-\left(1-\varepsilon^{\prime}\right)^{m} M \Delta\left(|\sigma|^{\frac{m}{1-m}} \zeta(\sigma)\right) \\
& \geq\left(1-\varepsilon^{\prime}\right)^{m} \Delta U^{m}-C\left[\left(r^{2}+\sigma^{2}\right)^{\frac{1}{2}} c^{-1} U^{-1}\right]\left(r^{2}+\sigma^{2}\right)^{-\frac{1}{2}} c U
\end{aligned}
$$

with a constant $C=C\left(\varepsilon^{\prime}, M, \zeta\right)>0$. Since (3.11) also holds for the case $-2 \leq \sigma \leq$ -1 , we see from $r^{2}+\sigma^{2} \leq 5$ and $|\sigma| \geq 1$ that

$$
\begin{aligned}
\left(r^{2}+\sigma^{2}\right)^{\frac{1}{2}} c^{-1} U^{-1} & =\left(r^{2}+\sigma^{2}\right)^{\frac{1}{2}} c^{\frac{1}{1-m}-1} A^{-1}\left(\sqrt{r^{2}+\sigma^{2}}+\sigma\right)^{\frac{1}{1-m}} \\
& \leq C\left(\sqrt{r^{2}+\sigma^{2}}+\sigma\right)^{\frac{1}{1-m}} \leq C\left(\frac{r_{0}^{2}}{|\sigma|}\right)^{\frac{1}{1-m}} \leq C r_{0}^{\frac{2}{1-m}}=O\left(r_{0}^{\frac{2}{1-m}}\right)
\end{aligned}
$$

as $r_{0} \rightarrow 0$, where $C=C(m, A, c)>0$ is some constant. Hence we obtain the same estimates as in (3.12). Then we see that $u^{-}$is a sub-solution in the case (3.9) with $-2 \leq \sigma \leq-1$ if $r_{0}$ is sufficiently small depending only on $m, n, A, c, K, M, \varepsilon^{\prime}$ and $\zeta$. 
Finally, we examine the case (3.9) with $-1 \leq \sigma \leq 1$. In this case, we have

$$
\begin{aligned}
& u_{t}^{-}=\left(1-\varepsilon^{\prime}\right) \frac{1}{m}\left[U^{m}-M\right]^{\frac{1}{m}-1} m U^{m-1} U_{t} \leq\left(1-\varepsilon^{\prime}\right) U_{t}, \\
& \Delta\left(u^{-}\right)^{m}=\left(1-\varepsilon^{\prime}\right)^{m} \Delta U^{m} .
\end{aligned}
$$

Then the same estimates as in (3.12) immediately follow, and so $u^{-}$is a sub-solution in the case (3.9) with $-1 \leq \sigma \leq 1$ if $r_{0}$ is sufficiently small depending only on $m$, $n, A, K$ and $\varepsilon^{\prime}$.

Recall that we only have to consider the case (3.9). Thus, $u^{-}$is a sub-solution of (1.1) on $Q$ provided that $r_{0}$ is sufficiently small depending only on $m, n, A, c$, $K, M, \varepsilon^{\prime}$ and $\zeta$.

3.5. Positive comparison functions. We prove Proposition 3.1.

Proof of Proposition Proposition 3.1. Let $0<\varepsilon^{\prime}<\varepsilon<1$. We set $\bar{u}$ and $u^{-}$as in (3.5) and (3.7), respectively. Define

$$
\underline{u}(x, t):=\max \left\{u^{-}(x, t), \varepsilon\right\} .
$$

Since the maximum of two sub-solutions is also a sub-solution, $\underline{u}$ is a positive subsolution on $Q$. Moreover, we can easily check that $\underline{u} \leq \bar{u}$ on $Q$. Then (i) holds.

We prove (ii). By the choice of $\bar{u}$ and $\underline{u}$, we have

$$
\begin{aligned}
& \bar{u}=\left[\left(1+\varepsilon^{\prime}\right)^{m}\left(U^{m}+1\right)+B(b-1)\right]^{\frac{1}{m}}, \\
& \underline{u}=\left(1-\varepsilon^{\prime}\right)\left[U^{m}-M-M|\sigma|^{\frac{m}{1-m}} \zeta(\sigma)\right]_{+}^{\frac{1}{m}}
\end{aligned}
$$

for $(x, t) \in \Gamma_{r_{1}} \backslash \Gamma$. For $(x, t) \in \mathbb{R}^{n+1}$ with $0<r(x) \leq \delta$ and $\sigma \leq \delta$, by (3.11), we have

$$
\begin{aligned}
U^{m} & =A^{m} c^{-\frac{m}{1-m}}\left(\sqrt{r^{2}+\sigma^{2}}+\sigma\right)^{-\frac{m}{1-m}} \\
& \geq \begin{cases}(\sqrt{2}+1)^{-\frac{m}{1-m}} A^{m} c^{-\frac{m}{1-m}} \delta^{-\frac{m}{1-m}} & \text { if }-\infty<\sigma \leq \delta, \\
A^{m} c^{-\frac{m}{1-m}}\left(\frac{\delta^{2}}{2|\sigma|}\right)^{-\frac{m}{1-m}} & \text { if }-\infty<\sigma<0 .\end{cases}
\end{aligned}
$$

Then there exists a constant $C=C(m, A, c)>0$ such that

$$
1 \leq \begin{cases}C \delta^{\frac{m}{1-m}} U^{m} & \text { if }-\infty<\sigma \leq \delta, \\ C|\sigma|^{-\frac{m}{1-m}} \delta^{\frac{2 m}{1-m}} U^{m} & \text { if }-\infty<\sigma<0 .\end{cases}
$$

From this, it follows that

$$
\bar{u} \leq\left[\left(1+\varepsilon^{\prime}\right)^{m}\left(1+C \delta^{\frac{m}{1-m}}\right)+C B(b-1) \delta^{\frac{m}{1-m}}\right]^{\frac{1}{m}} U \leq(1+\varepsilon) U
$$

and

$$
\begin{aligned}
\underline{u} & = \begin{cases}\left(1-\varepsilon^{\prime}\right)\left[U^{m}-M-M|\sigma|^{\frac{m}{1-m}} \zeta(\sigma)\right]_{+}^{\frac{1}{m}} & (-\infty<\sigma<0) \\
\left(1-\varepsilon^{\prime}\right)\left[U^{m}-M\right]_{+}^{\frac{1}{m}} & (0 \leq \sigma \leq \delta)\end{cases} \\
& \geq \begin{cases}\left(1-\varepsilon^{\prime}\right)\left[1-C M \delta^{\frac{m}{1-m}}-C M \delta^{\frac{2 m}{1-m}}\right]_{+}^{\frac{1}{m}} U & (-\infty<\sigma<0) \\
\left(1-\varepsilon^{\prime}\right)\left[1-M C \delta^{\frac{m}{1-m}}\right]_{+}^{\frac{1}{m}} U & (0 \leq \sigma \leq \delta)\end{cases} \\
& \geq(1-\varepsilon) U
\end{aligned}
$$


for $(x, t) \in \mathbb{R}^{n+1}$ with $0<r(x) \leq \delta$ and $-\infty<\sigma \leq \delta$ provided that $\delta$ is sufficiently small depending only on $m, A, b, B, c, M, \varepsilon$ and $\varepsilon^{\prime}$. Hence (ii) follows.

\section{Proof of the main theorem}

We define an exhaustion of $Q$, and then we apply an argument from [7] (see also [4, Lemma 2.1]) to show the existence of an entire-in-time singular solution.

Proof of Theorem 1.2. Let $\left\{\Omega_{i}(t)\right\}_{t \in \mathbb{R}}$ be a family of smooth bounded domains in $\mathbb{R}^{n}$ such that $\Omega_{i}(t) \subsetneq \Omega_{i+1}(t), \bigcup_{i>1} \Omega_{i}(t)=\mathbb{R}^{n} \backslash \Gamma(t)$ for each $t \in \mathbb{R}$ and $S_{i}:=$ $\left\{(x, t) \in \mathbb{R}^{n+1} ; x \in \partial \Omega_{i}(t), t \in(-i, i)\right\}$ is smooth. Define

$$
Q_{i}:=\left\{(x, t) \in \mathbb{R}^{n} ; x \in \Omega_{i}(t), t \in(-i, i)\right\} .
$$

Note that $\bigcup_{i \geq 1} Q_{i}=Q$ and $Q_{i} \subsetneq Q_{i+1}$. Consider the following approximate problem.

$$
\begin{cases}w_{t}=\Delta w^{m} & \text { in } Q_{i}, \\ w=\underline{u} & \text { on } S_{i}, \\ w(\cdot,-i)=\underline{u}(\cdot,-i) & \text { in } \Omega_{i}(-i) .\end{cases}
$$

By the assumptions on $\Omega_{i}$ and the uniform positivity of comparison functions $\bar{u}$ and $\underline{u}$ on $Q_{i}$, this approximate problem has a bounded solution $w_{i}$ satisfying $\underline{u} \leq w_{i} \leq \bar{u}$ in $Q_{i}$. Since $\underline{u}$ is a sub-solution, the comparison principle for bounded solutions implies that $w_{i}(\cdot,-i)=\underline{u}(\cdot,-i) \leq w_{i+1}(\cdot,-i)$ in $\Omega_{i}(-i)$. From the comparison principle for bounded solutions again, it follows that

$$
\underline{u} \leq w_{i} \leq w_{i+1} \leq \bar{u} \quad \text { in } Q_{i}
$$

for each $i$. Hence the limiting function

$$
u(x, t):=\lim _{i \rightarrow \infty} w_{i}(x, t), \quad(x, t) \in Q
$$

exists and satisfies $\underline{u} \leq u \leq \bar{u}$ in $Q$. By the same argument as in [6, Lemma 5.1] based on the parabolic interior regularity theory and a diagonalization argument, we see that $u \in C^{2,1}(Q)$ and $w_{i} \rightarrow u$ in $C_{\mathrm{loc}}^{2,1}(Q)$ as $i \rightarrow \infty$. Hence $u$ satisfies (1.1) in $Q$. Moreover, the desired estimate on $u$ immediately follows from Proposition 3.1 , and the proof is complete.

\section{DisCUSSION}

While our focus here is on furthering the classification of singular behavior in fast nonlinear diffusion, rather than upon applications, we now briefly comment on the nature of latter that lies in the background. A key phenomenon associated with fast diffusion is the suppression of transport at high "concentrations" $u$ (see [18] for a number of illustrative applications). It is hoped that the associated intuition clarifies the physical status of singular solutions in the context of localized sources of material, the line singularities in the above being associated with restricted ability of material to diffuse away from the ridge of high concentration laid down by the moving source at the head of the snake (possibly augmented by continued injection along the evolving line).

The snaking solutions are the simplest representatives of a much more general class, that is in turn illustrative of very wide-ranging issues of non-uniqueness in the equation of fast diffusion (cf. [6] and references therein), whereby the head of the snake can be specified to take any path leaving in its wake a line singularity. 
Before we give a formal argument for this, we introduce a transformed equation and some notation. Writing $W:=m u^{m-1}$ takes equation (1.1) to the quadratically nonlinear form

$$
W_{t}=W \Delta W-\frac{1}{1-m}|\nabla W|^{2} .
$$

We use the notation $x=\left(x_{1}, x_{2}, \ldots, x_{n}\right) \in \mathbb{R}^{n}, \rho:=\left(x_{2}^{2}+\cdots+x_{n}^{2}\right)^{1 / 2}$. A formal argument proceeds along the following lines: at a point on which the singular curve is smooth, we take the $x_{1}$ direction to be tangential to the curve, the dominant balance then reading

$$
W_{t} \sim W\left(W_{\rho \rho}+\frac{n-2}{\rho} W_{\rho}\right)-\frac{1}{1-m} W_{\rho}^{2}
$$

on the assumption of cylindrical symmetry, this having a self-consistent local solution

$$
W \sim \frac{\rho^{2}}{2 B\left(t-t_{*}\right)}, \quad B:=\frac{n-1}{1-m}\left(m-\frac{n-3}{n-1}\right), \quad \text { as } \rho \rightarrow 0, t>t_{*},
$$

where $t_{*}$ is the time at which the head of the snake passes through the location in question, the behavior at the head being a quasi-steady generalization of the above traveling wave solution.

Numerous natural generalizations presumably arise: the head of the snake can come to a halt or retreat, its path may not need to be smooth and singular sets of dimensionality greater than one are possible (the simplest such examples being the above solutions embedded in higher dimensional space with no dependence on the additional dimensions). Very specific questions relate to whether cylindrical symmetry necessarily follows in the sense of (5.2)-(5.3) and with respect to the large-time behavior when the head comes to a stop in finite time.

\section{Appendix A. The traveling WaVe SOlution}

We seek a traveling wave for (5.1) in the $x_{1}$ direction, writing

$$
w=W\left(x_{1}-t a, x_{2}, \ldots, x_{n}\right), \quad a>0
$$

and introducing paraboloidal coordinates $Y$ and $Z$ via

$$
x_{1}-t a=\frac{1}{2}\left(Y^{2}-Z^{2}\right), \quad R=Y Z, \quad W=\phi(Y, Z)
$$

to give

$$
\begin{aligned}
-a\left(Y \phi_{Y}-Z \phi_{Z}\right)= & \phi\left(\phi_{Y Y}+\phi_{Z Z}+(n-2)\left(\frac{1}{Y} \phi_{Y}+\frac{1}{Z} \phi_{Z}\right)\right) \\
& -\frac{1}{1-m}\left(\phi_{Y}^{2}+\phi_{Z}^{2}\right) .
\end{aligned}
$$

Setting $\phi=\phi(Y)$ yields the ODE

$$
-a Y \phi_{Y}=\phi\left(\phi_{Y Y}+\frac{n-2}{Y} \phi_{Y}\right)-\frac{1}{1-m} \phi_{Y}^{2}
$$

that corresponds to the Boltzmann similarity reduction of the porous-medium equation in $n-1$ dimensions and whose scaling properties imply the existence of a solution $\phi=a Y^{2} / B$ equivalent to the representation (2.2)-(2.3). This solution thus 
constitutes a reduction of (1.1) akin to that exploited by the Ivantsov solution to the Stefan problem.

\section{ACKNOWLEDGMENTS}

The authors express their gratitude to Dr. Takashi Kagaya for his valuable comments concerning the regularity of the distance function and to the referee for useful suggestions.

\section{REFERENCES}

[1] E. Chasseigne, Classification of razor blades to the filtration equation-the sublinear case, J. Differential Equations 187 (2003), no. 1, 72-105, DOI 10.1016/S0022-0396(02)00019-0. MR1946547

[2] E. Chasseigne and J. L. Vazquez, Theory of extended solutions for fast-diffusion equations in optimal classes of data. Radiation from singularities, Arch. Ration. Mech. Anal. 164 (2002), no. 2, 133-187, DOI 10.1007/s00205-002-0210-0. MR1929929

[3] E. Chasseigne and J. L. Vázquez, The pressure equation in the fast diffusion range, Rev. Mat. Iberoamericana 19 (2003), no. 3, 873-917, DOI 10.4171/RMI/373. MR2053567

[4] X. Chen and J.-S. Guo, Existence and uniqueness of entire solutions for a reaction-diffusion equation, J. Differential Equations 212 (2005), no. 1, 62-84, DOI 10.1016/j.jde.2004.10.028. MR2130547

[5] M. Fila, P. Macková, J. Takahashi, and E. Yanagida, Moving singularities for nonlinear diffusion equations in two space dimensions, J. Elliptic Parabol. Equ. 6 (2020), no. 1, 155169, DOI 10.1007/s41808-020-00062-0. MR4114347

[6] M. Fila, J. Takahashi, and E. Yanagida, Solutions with moving singularities for equations of porous medium type, Nonlinear Anal. 179 (2019), 237-253, DOI 10.1016/j.na.2018.08.016. MR3886631

[7] Y. Fukao, Y. Morita, and H. Ninomiya, Some entire solutions of the Allen-Cahn equation, Proceedings of Third East Asia Partial Differential Equation Conference, 2004, pp. 15-32, DOI 10.11650/twjm/1500558454. MR2057634

[8] B. H. Gilding and R. Kersner, Travelling waves in nonlinear diffusion-convection reaction, Progress in Nonlinear Differential Equations and their Applications, vol. 60, Birkhäuser Verlag, Basel, 2004. MR2081104

[9] M. A. Herrero and M. Pierre, The Cauchy problem for $u_{t}=\Delta u^{m}$ when $0<m<1$, Trans. Amer. Math. Soc. 291 (1985), no. 1, 145-158, DOI 10.2307/1999900. MR797051

[10] K. P. P. Htoo, J. Takahashi, and E. Yanagida, Higher-dimensional moving singularities in a superlinear parabolic equation, J. Evol. Equ. 18 (2018), no. 4, 1575-1593, DOI 10.1007/s00028-018-0452-4. MR3884761

[11] K. P. P. Htoo and E. Yanagida, Singular solutions of a superlinear parabolic equation with homogeneous Neumann boundary conditions, Nonlinear Anal. 151 (2017), 96-108, DOI 10.1016/j.na.2016.11.015. MR3596672

[12] K. M. Hui and S. Kim, Asymptotic large time behavior of singular solutions of the fast diffusion equation, Discrete Contin. Dyn. Syst. 37 (2017), no. 11, 5943-5977, DOI 10.3934/dcds.2017258. MR3681967

[13] T. Kan and J. Takahashi, On the profile of solutions with time-dependent singularities for the heat equation, Kodai Math. J. 37 (2014), no. 3, 568-585, DOI 10.2996/kmj/1414674609. MR3273884

[14] _ , Time-dependent singularities in semilinear parabolic equations: behavior at the singularities, J. Differential Equations 260 (2016), no. 10, 7278-7319, DOI 10.1016/j.jde.2016.01.026. MR3473442

[15] _ , Time-dependent singularities in semilinear parabolic equations: existence of solutions, J. Differential Equations 263 (2017), no. 10, 6384-6426, DOI 10.1016/j.jde.2017.07.016. MR3693178

[16] G. Karch, M. E. Schonbek, and T. P. Schonbek, Singularities of certain finite energy solutions to the Navier-Stokes system, Discrete Contin. Dyn. Syst. 40 (2020), no. 1, 189-206, DOI 10.3934/dcds.2020008. MR4026957 
[17] G. Karch and X. Zheng, Time-dependent singularities in the Navier-Stokes system, Discrete Contin. Dyn. Syst. 35 (2015), no. 7, 3039-3057, DOI 10.3934/dcds.2015.35.3039. MR3343553

[18] J. R. King, Self-similar behaviour for the equation of fast nonlinear diffusion, Philos. Trans. Roy. Soc. London Ser. A 343 (1993), 337-375, DOI 10.1098/rsta.1993.0052.

[19] S. Sato and E. Yanagida, Solutions with moving singularities for a semilinear parabolic equation, J. Differential Equations 246 (2009), no. 2, 724-748, DOI 10.1016/j.jde.2008.09.004. MR2468735

[20], Forward self-similar solution with a moving singularity for a semilinear parabolic equation, Discrete Contin. Dyn. Syst. 26 (2010), no. 1, 313-331, DOI 10.3934/dcds.2010.26.313. MR2552790

[21] _ Singular backward self-similar solutions of a semilinear parabolic equation, Discrete Contin. Dyn. Syst. Ser. S 4 (2011), no. 4, 897-906, DOI 10.3934/dcdss.2011.4.897. MR2746450

[22] J. Takahashi, Existence of solutions with moving singularities for a semilinear heat equation with a critical exponent, J. Math. Pures Appl. (9) 148 (2021), 128-149, DOI 10.1016/j.matpur.2021.02.007. MR4223350

[23] J. Takahashi and H. Yamamoto, Solutions with time-dependent singular sets for the heat equation with absorption, J. Differential Equations 266 (2019), no. 7, 4061-4105, DOI 10.1016/j.jde.2018.09.029. MR3912711

[24] J. Takahashi and E. Yanagida, Time-dependent singularities in the heat equation, Commun. Pure Appl. Anal. 14 (2015), no. 3, 969-979, DOI 10.3934/cpaa.2015.14.969. MR3320161

[25] _ , Time-dependent singularities in a semilinear parabolic equation with absorption, Commun. Contemp. Math. 18 (2016), no. 5, 1550077, 27, DOI 10.1142/S0219199715500777. MR3523183

[26] J. L. Vázquez, Smoothing and decay estimates for nonlinear diffusion equations, Oxford Lecture Series in Mathematics and its Applications, vol. 33, Oxford University Press, Oxford, 2006. MR2282669

[27] J. L. Vázquez and M. Winkler, The evolution of singularities in fast diffusion equations: infinite-time blow-down, SIAM J. Math. Anal. 43 (2011), no. 4, 1499-1535, DOI 10.1137/100809465. MR2821594

Department of Applied Mathematics and Statistics, Comenius University, 84248 Bratislava, Slovakia

E-mail address, Corresponding author: fila@fmph.uniba.sk

Theoretical Mechanics Section, University of Nottingham, Nottingham NG7 2RD, $\mathrm{UK}$

E-mail address: john.king@nottingham.ac.uk

Department of Mathematical and Computing Science, Tokyo Institute of TechnolOGY, TOKYO 152-8552, JAPAN

E-mail address: takahashi@c.titech.ac.jp

Department of Mathematics, Tokyo Institute of Technology, Tokyo 152-8551, Japan

E-mail address: yanagida@math.titech.ac.jp 